\title{
ARTICLE
}

\section{Interstitial oxygen as a source of $p$-type conductivity in hexagonal manganites}

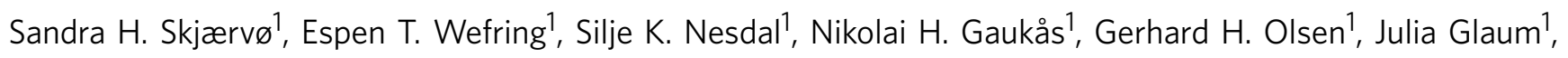
Thomas Tybell ${ }^{2} \&$ Sverre M. Selbach ${ }^{1}$

Hexagonal manganites, $\mathrm{h}-R \mathrm{MnO}_{3}(R=\mathrm{Sc}, \mathrm{Y}, \mathrm{Ho}-\mathrm{Lu})$, have been intensively studied for their multiferroic properties, magnetoelectric coupling, topological defects and electrically conducting domain walls. Although point defects strongly affect the conductivity of transition metal oxides, the defect chemistry of $\mathrm{h}-\mathrm{RMnO}_{3}$ has received little attention. We use a combination of experiments and first principles electronic structure calculations to elucidate the effect of interstitial oxygen anions, $\mathrm{O}_{\mathrm{i}}$, on the electrical and structural properties of $\mathrm{h}-\mathrm{YMnO}_{3}$. Enthalpy stabilized interstitial oxygen anions are shown to be the main source of p-type electronic conductivity, without reducing the spontaneous ferroelectric polarization. A low energy barrier interstitialcy mechanism is inferred from Density Functional Theory calculations to be the microscopic migration path of $\mathrm{O}_{i}$. Since the $\mathrm{O}_{i}$ content governs the concentration of charge carrier holes, controlling the thermal and atmospheric history provides a simple and fully reversible way of tuning the electrical properties of $\mathrm{h}-\mathrm{RMnO}_{3}$.

\footnotetext{
${ }^{1}$ Department of Materials Science and Engineering, NTNU Norwegian University of Science and Technology, NO-7491 Trondheim, Norway. ${ }^{2}$ Department of Electronics and Telecommunications, NTNU Norwegian University of Science and Technology, NO-7491 Trondheim, Norway. Correspondence and requests for materials should be addressed to S.M.S. (email: selbach@ntnu.no).
} 
P oint defects are imperative to the functional properties of oxides used in electrochemical devices like solid oxide fuel cells, batteries and memristors ${ }^{1-3}$. In contrast, point defects in general have a detrimental effect on physical properties of oxides for electronics, such as for example fatigue and domain wall pinning in ferroelectrics ${ }^{4}$. As components are made smaller, the available length for point defects in materials to diffuse is made shorter. This reduces the time required for the point defect to diffuse through the samples compared with bulk materials, where they effectively freeze in ${ }^{5}$. Understanding point defects in functional oxides thus becomes ever more important with decreasing component dimensions ${ }^{6,7}$.

Rare earth ternary manganites with $\mathrm{RMnO}_{3}$ stoichiometry are stable in the hexagonal manganite structure with space group $P 6_{3} \mathrm{~cm}$ (185) for $R=\mathrm{Sc}, \mathrm{Y}$ and Ho-Lu. The structure consists of layers of five-coordinated $\mathrm{Mn}^{3+}$ corner-sharing trigonal bipyramids separated by layers of $\mathrm{Y}^{3+}$ in the $a b$-plane. The $\mathrm{MnO}_{5}$ bipyramids are tilted in a pattern of trimers, while the $\mathrm{Y}^{3+}$ are displaced in opposite directions along the polar $c$-axis ${ }^{8}$. A subtle shift of the trigonal bipyramid layer with respect to the $\mathrm{Y}^{3+}$ layer, caused by an improper ferroelectric transition at $1,250 \mathrm{~K}$, is the origin of the ferroelectric polarization ${ }^{9}$. The $\mathrm{Mn}^{3+}$ sublattice has a frustrated non-collinear antiferromagnetic order on a trigonal lattice, with a Néel temperature of $75 \mathrm{~K}$ (ref. 10).

Compared with the ternary $\mathrm{ABO}_{3}$ perovskite oxides, the defect chemistry of $\mathrm{h}-\mathrm{RMnO}_{3}$ has received little attention. Oxygen vacancies in $\mathrm{YMnO}_{3}$ have been studied ${ }^{11-13}$, and large oxygen excess has been reported for h- $R \mathrm{MnO}_{3+\delta}(R=\mathrm{Y}$, Dy) with $\delta$ up to 0.35 for $R=\mathrm{Dy}^{14}$. High levels of excess oxygen can cause development of secondary oxidized phases, which are easily detected experimentally ${ }^{15}$. However, structural effects, energetics and kinetics of point defects in low concentrations have not been addressed. Excess oxygen is well known in perovskite manganites such as $\mathrm{LaMnO}_{3+\delta}$. Since the perovskite structure is too closepacked for interstitial oxygen anions, excess oxygen is accommodated by cation vacancies; $\mathrm{La}_{1-\delta / 3} \mathrm{Mn}_{1-\delta / 3} \mathrm{O}_{3}$ (ref. 16). Oxidation of perovskite $\mathrm{LaMnO}_{3}$ thus requires cation diffusion, which demands higher temperatures than anion diffusion.

Diffusion of oxygen in bulk $\mathrm{YMnO}_{3}$ has been observed below $200^{\circ} \mathrm{C}$ (ref. 14), making cation vacancy compensation highly unlikely. The layered $\mathrm{h}-\mathrm{R} \mathrm{MnO}_{3}$ structure is however $\sim 11 \%$ less dense than the corresponding orthorhombic perovskite structure, suggesting the possibility of interstitial oxygen. This is further supported by the observation that crystallization, which requires cation diffusion, of amorphous $\mathrm{YMnO}_{3}$ only occurs above $800^{\circ} \mathrm{C}$ (ref. 17).

Here we investigate how excess oxygen in the form of highly mobile interstitial anions is accommodated in the hexagonal manganite structure, and demonstrate that this enthalpy stabilized point defect gives rise to the observed p-type conductivity in $\mathrm{YMnO}_{3}$. This shows the importance of controlling the material's thermo-atmospheric history and opens a new avenue for tuning the physical properties of hexagonal manganites.

\section{Results}

Oxygen stoichiometry and electrical conductivity. First we consider the effect of thermal and atmospheric history on the conductivity and oxygen stoichiometry of the prototypical hexagonal manganite $\mathrm{YMnO}_{3}$. Thermoelectric power for a porous polycrystalline $\mathrm{YMnO}_{3}$ bar was measured in $\mathrm{O}_{2}$ and $\mathrm{N}_{2}$ atmosphere, Fig. 1a. The Seebeck coefficient is positive for all temperatures in $\mathrm{O}_{2}$ atmosphere, implying that $p$-type electronic conduction dominates, and a maximum is observed at $300^{\circ} \mathrm{C}$. In
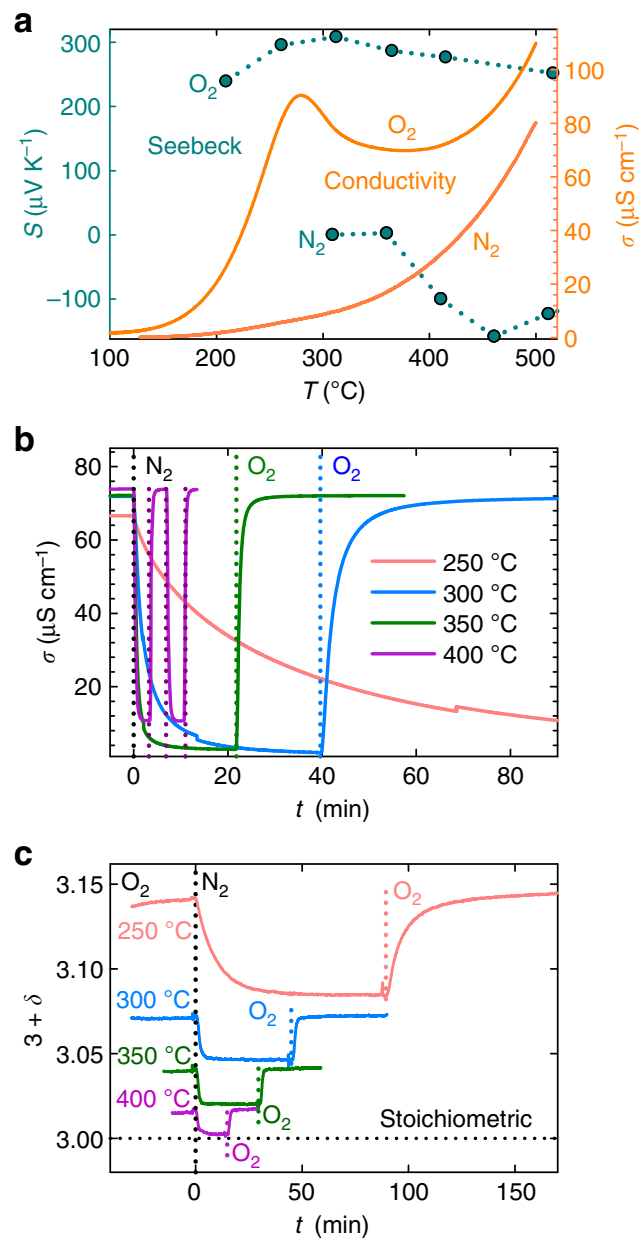

Figure 1 | p-type conductivity and excess oxygen. (a) Seebeck coefficient, $\mathrm{S}$, and DC electrical conductivity, $\sigma$, in $\mathrm{O}_{2}$ and $\mathrm{N}_{2}$ atmosphere of a porous polycrystal of $\mathrm{YMnO}_{3}$, as a function of temperature, T. (b) DC electrical conductivity as a function of time, $t$, of a porous polycrystal upon switching atmosphere (indicated by dotted lines) between flowing $\mathrm{O}_{2}$ and $\mathrm{N}_{2}$. The atmosphere is switched twice between $\mathrm{O}_{2}$ and $\mathrm{N}_{2}$ at $400^{\circ} \mathrm{C}$. (c) Oxygen stoichiometry, $3+\delta$, as a function of time, $t$, upon switching atmosphere measured by thermogravimetry for $\mathrm{YMnO}_{3}$ nanoparticles $\left(d_{X R D}=49 \pm 4 n m\right)$.

$\mathrm{N}_{2}$ atmosphere, the Seebeck coefficient is negative, implying conduction by electrons as majority charge carriers. The DC electrical conductivity of $\mathrm{YMnO}_{3}$ in $\mathrm{O}_{2}$ increases exponentially on heating from $100^{\circ} \mathrm{C}$, as expected for a semiconducting oxide. However, the conductivity goes through a maximum value at $270^{\circ} \mathrm{C}$, then decreases and stays relatively constant up to $420^{\circ} \mathrm{C}$, from which the conductivity again increases exponentially. In $\mathrm{N}_{2}$ atmosphere, the DC conductivity follows an exponential trend on heating, as expected for a semiconductor.

The observations in Fig. 1a is consistent with the chemical defect reaction:

$$
1 / 2 \mathrm{O}_{2}(\mathrm{~g})+2 \mathrm{Mn}_{\mathrm{Mn}}^{\mathrm{x}} \stackrel{\mathrm{YMnO}_{3}}{\longrightarrow} 2 \mathrm{Mn}_{\mathrm{Mn}}^{\bullet}+\mathrm{O}_{\mathrm{i}}^{\prime \prime}
$$

where $\mathrm{O}_{\mathrm{i}}^{\prime \prime}$ is interstitial $\mathrm{O}^{2-}$, and $\mathrm{Mn}_{\mathrm{Mn}}^{\mathrm{x}}$ and $\mathrm{Mn}_{\mathrm{Mn}}^{\bullet}$ depicts $\mathrm{Mn}^{3+}$ and $\mathrm{Mn}^{4+}$ on Mn lattice sites, respectively. Tetravalent $\mathrm{Mn}$ in oxidized $\mathrm{YMnO}_{3}$ is $\mathrm{Mn}$ with localized electron holes: $\mathrm{Mn}^{4+}=\mathrm{Mn}^{3+}+h^{+}$. Holes as the majority charge carrier is consistent with the positive sign of the Seebeck coefficient. The mobility of negatively charged interstitial $\mathrm{O}^{2-}$ is expected to be 
orders of magnitude smaller than for holes, and this is addressed further below.

The peculiar thermal evolution of the electrical conductivity can be explained from the maximum oxygen stoichiometry observed at about $250^{\circ} \mathrm{C}$ (ref. 14), which coincides with a maximum in electrical conductivity. The deviation in conductivity from the exponential behaviour of a semiconductor stems from the different temperature dependence of the concentration and mobility of holes. While the mobility of holes increases with temperature, this is counterbalanced by the loss of oxygen and charge compensating holes, above $250^{\circ} \mathrm{C}$, as we will show further below. The atypical thermal evolution of the electrical properties of $\mathrm{YMnO}_{3}$ in this temperature range has previously been attributed to the filling of oxygen vacancies ${ }^{18-20}$, but charge compensating electrons would give a negative Seebeck coefficient.

DC conductivity measurements were then performed on isothermal change of atmospheres between $\mathrm{O}_{2}$ and $\mathrm{N}_{2}$. When the partial pressure of oxygen, $p \mathrm{O}_{2}$, is reduced by switching from $\mathrm{O}_{2}$ to $\mathrm{N}_{2}$ atmosphere, the oxygen excess decreases according to equation (1) and a relaxation towards a lower conductivity is observed in Fig. 1b. The initial conductivity is regained after changing the atmosphere back to $\mathrm{O}_{2}$, demonstrating the reversibility of reaction (1). The regained conductivity is similar for all isotherms, owing to the opposing effects of decreasing charge carrier mobility and increasing concentration as the temperature is reduced. The relaxation time before equilibrium is reached is highly temperature dependent, as expected for diffusion of oxygen into the lattice.

AC conductivity measurements were performed on samples annealed in flowing $\mathrm{O}_{2}$ and $\mathrm{N}_{2}$, respectively, before measurement (Supplementary Fig. 1). The conductivity of the $\mathrm{O}_{2}$ annealed sample shows the characteristic maximum at $280^{\circ} \mathrm{C}$ on heating, resembling the maximum observed in Fig. 1a. The conductivity measured on cooling is lower compared with the initial heating cycle reflecting the loss of $\mathrm{O}_{\mathrm{i}}$ that occurred at higher temperatures. The $\mathrm{N}_{2}$ annealed sample exhibits a smooth increase of the conductivity with temperature. Subsequent cooling leads to higher conductivity in accordance with the reversibility of the $\mathrm{O}_{i}$ incorporation process.

The reversibility of reaction (1) is also evidenced by the oxygen stoichiometry when switching between $\mathrm{O}_{2}$ and $\mathrm{N}_{2}$ atmospheres, Fig. 1c. As the length scale of the system is imperative to diffusion controlled processes ${ }^{5}$, nanoparticles with an average crystallite size of $d_{\mathrm{XRD}}=49 \pm 4 \mathrm{~nm}$ (ref. 17) were chosen for studying thermogravimetric relaxation on switching atmosphere at a shorter time-scale than in bulk material. With increasing temperature the oxygen stoichiometry equilibrates faster. The rapid change in oxygen stoichiometry in $\mathrm{YMnO}_{3}$ nanoparticles illustrates the increasing importance of thermal and atmospheric history with decreasing system size. The isothermal response of the electrical conductivity (Fig. 1b) and the oxygen stoichiometry (Fig. 1c) on change in $p \mathrm{O}_{2}$ is explained by reaction (1): oxygen hyperstoichiometry governs the electrical conductivity of h- $R \mathrm{MnO}_{3+\delta}$.

Position of interstitial oxygen and structural effects. The low temperature at which $\mathrm{YMnO}_{3}$ exchanges oxygen with the atmosphere makes cation vacancies and diffusion unrealistic, and the positive Seebeck coefficient from mobile holes points to interstitial oxygen anions, $\mathrm{O}_{\mathrm{i}}$, as the dominating point defect. Hightemperature X-ray diffraction measurements of nanocrystalline $\mathrm{YMnO}_{3}$ show that interstitial oxygen is incorporated into the lattice, causing anisotropic chemical expansion (Supplementary Fig. 2). We now turn our attention to the position of interstitial oxygen in $\mathrm{YMnO}_{3}$. Potential energy surfaces (PES) were determined by mapping the energy landscape of the $\mathrm{O}_{\mathrm{i}}$ position in several lattice planes by static Density Functional Theory (DFT) calculations (See details in Supplementary Fig. 3 and Supplementary Note 1). For illustration, the PES of $\mathrm{O}_{\mathrm{i}}$ in the (002) (a) and $(3 / 200)$ (b) planes are included in Fig. 2 along wth a unit cell with the corresponding lattice planes (d). The relative energy of $\mathrm{O}_{\mathrm{i}}$ along the grey line in Fig. $2 \mathrm{~b}$ is shown in Fig. 2c. The most stable positions of $\mathrm{O}_{\mathrm{i}}$ was found to be between three $\mathrm{Mn}$ in the $\mathrm{Mn}-\mathrm{O}$ planes at $\mathrm{z}=0$ and $1 / 2$, resulting in six equivalent possible sites for $\mathrm{O}_{\mathrm{i}}$ in the $P 6_{3} \mathrm{~cm}$ unit cell, $(1 / 3,1 / 3,0),(2 / 3,0,0)$, $(0,2 / 3,0),(2 / 3,2 / 3,1 / 2),(1 / 3,0,1 / 2)$ and $(0,1 / 3,1 / 2)$, as illustrated by green circles in panels $\mathbf{a}$ and $\mathbf{b}$.

Experimental observations of a maximum $\delta$ of $\sim 0.35$ (refs 14,15) can be rationalized from our DFT simulations. If two out of the six possible stable interstitial sites in the unit cell are occupied, a hypothetical fully oxidized structure of $\mathrm{YMnO}_{3}$ would have a chemical formula of $\mathrm{YMnO}_{3.33}$ or $\mathrm{Y}_{3} \mathrm{Mn}_{3} \mathrm{O}_{10}$. This will result in one $\mathrm{O}_{i}$ and two out of three oxidized $\mathrm{Mn}$ in each $\mathrm{Mn}-\mathrm{O}$ layer of the 30 atom unit cell. Accommodation of more than two $\mathrm{O}_{i}$ per unit cell would require charge compensation across layers or further oxidation of $\mathrm{Mn}^{4+}$ to $\mathrm{Mn}^{5+}$ within the same layer.

The in-plane distances between $\mathrm{O}_{\mathrm{i}}$ and $\mathrm{Mn}$ after structural relaxation depicted in Fig. 3a,b show that there are two shorter and one longer $\mathrm{Mn}-\mathrm{O}_{i}$ bond. $\mathrm{O}_{i}$ is displaced towards two $d^{3} \mathrm{Mn}^{4+}$ ions (with a calculated magnetic moment of $3.06 \mu_{\mathrm{B}}$ ), and away from one $d^{4} \mathrm{Mn}^{3+}$ ion $\left(3.74 \mu_{\mathrm{B}}\right)$. The localization of holes on the two oxidized $\mathrm{Mn}$ ions gives three possible configurations of $\mathrm{O}_{\mathrm{i}}$ and its three surrounding $\mathrm{Mn}$ ions. The most stable positions of $\mathrm{O}_{\mathrm{i}}$ are triple wells due to asymmetric electrostatic attraction: $\mathrm{O}_{i}$ with formal charge -2 has shorter bonds to the two $\mathrm{Mn}^{4+}$ than to the single $\mathrm{Mn}^{3+}$. Details and discussion about the energetic asymmetry of this triple well and the effect of $\mathrm{O}_{i}$ on the magnetism can be found in Supplementary Figs 4 and 5 and Supplementary Note 2.

Localization of holes on $\mathrm{Mn}$, and the resulting $p$-type polaronic conduction, stems from electrostatic attraction between positive holes and negative interstitial anions. In contrast, hole doping by substituting $\mathrm{Y}^{3+}$ with $\mathrm{Ca}^{2+}$ gives holes in Bloch states, while electron doping by $\mathrm{Zr}^{4+}$ for $\mathrm{Y}^{3+}$ substitution gives a polaronic state $^{21}$. The energy barrier for moving the holes associated with $\mathrm{O}_{\mathrm{i}}$ between different $\mathrm{Mn}^{4+}$ pairs is $0.29 \mathrm{eV}$ from nudged-elastic band (NEB) calculations (See details in Supplementary Fig. 6 and Supplementary Note 3). Our calculated energy barrier is lower than the reported activation energy of $0.38-0.50 \mathrm{eV}$ for polaron hopping in $\mathrm{YMnO}_{3}$ (refs 22-24).

Interstitial oxygen causes only subtle local structural distortions, as evident from the excerpt of the (002) plane of $\mathrm{YMnO}_{3}$ in Fig. 3a. The relaxed atomic positions are shown in the foreground, while the atoms in a perfect crystal are included in faded colours in the background for comparison. The Y sublattice is virtually unaffected by the introduction of $\mathrm{O}_{\mathrm{i}}$ in the $\mathrm{Mn}-\mathrm{O}$ layer, as shown in Fig. 3c. The local displacements of ions occur mainly within the same layer of trigonal $\mathrm{MnO}_{5}$ bipyramids as $\mathrm{O}_{\mathrm{i}}$ is situated, and the distortions decrease rapidly with increasing distance from $\mathrm{O}_{\mathrm{i}}$. The apical tilting angle, defined in Fig. 3d, of the three bipyramids surrounding $\mathrm{O}_{\mathrm{i}}$ is reduced from $8.7^{\circ}$ in the perfect crystal to $6.4^{\circ}$ for $\mathrm{Mn}^{4+}$ bipyramids and $4.7^{\circ}$ for $\mathrm{Mn}^{3+}$ bipyramids, respectively.

The local structural distortions caused by the introduction of $\mathrm{O}_{\mathrm{i}}$ can be quantified by the displacements of ions relative to the perfect $P 6_{3} \mathrm{~cm}$ structure as a function of the distance from the $\mathrm{O}_{\mathrm{i}}$, as illustrated in Fig. 3e. Displacements of Mn and planar oxygen closest to $\mathrm{O}_{i}$ are substantial, apical oxygen in the nearest bipyramids are also displaced, while $Y$ even close to $O_{i}$ are less affected. The structural screening length of the $\mathrm{O}_{\mathrm{i}}$ point defect is defined here as the distance away from $\mathrm{O}_{\mathrm{i}}$ where the displacement 

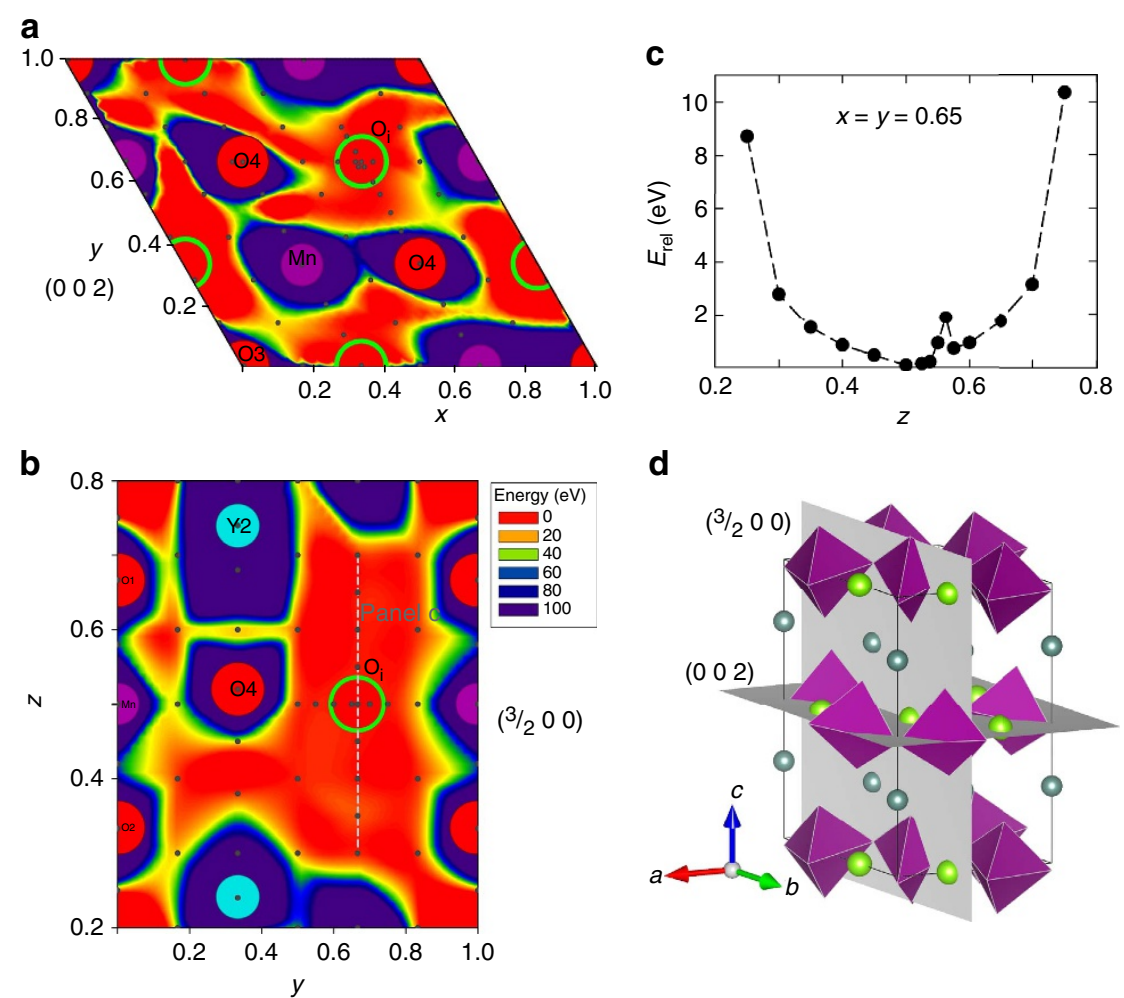

Figure 2 | Position of interstitial oxygen. Potential energy surfaces (PES) showing the energy landscape for the position of interstitial oxygen in a neutral $\mathrm{YMnO}_{3}$ unit cell in (a) the (002) plane and (b) the $(3 / 200)$ plane. (c) Relative energy along the $z$ direction for $x=y=0.65$ indicated by a light grey line in panel $\mathbf{b}$. The anomaly at $z=0.56$ stems from that the PES mapping was done with static calculations. (d) Crystal structure of $\mathrm{YMnO}_{3}$ showing purple $\mathrm{MnO}_{5}$ polyhedra, turquoise $\mathrm{Y}$ atoms, and green spheres marking the six equivalent stable positions for $\mathrm{O}_{\mathrm{i}}$ in the unit cell along with the two crystal planes in (a),(b).

is less than $0.1 \AA$ relative to the perfect structure. A structural screening length can thus be estimated to $5.5 \AA$, corresponding to the second coordination shell of $\mathrm{O}_{\mathrm{i}}$, and is shown by a dotted line in Fig. 3e. Local charge compensation of $\mathrm{O}_{\mathrm{i}}$ by two $\mathrm{Mn}^{4+}$ and polyhedral tilting mitigates the effect of $\mathrm{O}_{i}$ on the lattice. The short structural screening length implies that direct experimental detection of $\mathrm{O}_{\mathrm{i}}$ is challenging.

The calculations also show that the non-collinear triangular magnetic order is only subtly affected by $\mathrm{O}_{\mathrm{i}}$ (Supplementary Fig. 5). At the relevant temperatures for $\mathrm{O}_{i}$ transport, the material is paramagnetic and the properties are therefore not expected to be significantly affected by magnetic order. The relaxed structure is not affected by substituting the true non-collinear magnetic ground state structure with a synthetic collinear magnetic order.

Energetics of $\mathbf{O}_{\mathbf{i}}$. An essential thermodynamic quantity for point defects is the energy of formation. Using the formalism of Zhang et al. ${ }^{25}$ for neutral cells we can define the energy of formation, $E^{\mathrm{f}}$, for interstitial oxygen as:

$$
E_{\mathrm{O}_{\mathrm{i}}}^{\mathrm{f}}=E_{\mathrm{YMnO}_{3+\delta}}-E_{\mathrm{YMnO}_{3}}-\mu_{\mathrm{O}},
$$

where $E_{\mathrm{YMnO}_{3+\delta}}$ and $E_{\mathrm{YMnO}_{3}}$ are the total energies of oxidized and stoichiometric $\mathrm{YMnO}_{3}$, respectively, and $\mu_{\mathrm{O}}$ is the chemical potential of oxygen. The formation energy for $\mathrm{O}_{\mathrm{i}}$ in $\mathrm{YMnO}_{3}$ as a function of the chemical potential of oxygen is shown in Fig. 4a (See Supplementary Fig. 7 and Supplementary Methods for details on the thermodynamic stability region for bulk $\mathrm{YMnO}_{3}$ ). For $p_{\mathrm{O}_{2}}=0.21$, corresponding to air, the chemical potential of oxygen yields a negative formation energy at temperatures up to $\sim 900^{\circ} \mathrm{C}$, which is well above the temperature were $\mathrm{O}_{\mathrm{i}}$ becomes entropy destabilized, as seen from the experimental measurements in Fig. 1. However, it must be pointed out that the formation enthalpy for $\mathrm{O}_{i}$ is calculated in the dilute limit, while a finite concentration of $\mathrm{O}_{\mathrm{i}}$ is necessary to detect mass changes by thermogravimetry. The consumption of the gaseous species $\mathrm{O}_{2}(\mathrm{~g})$ in reaction (1) means that the entropy of this reaction is negative, hence the entropy contribution to the Gibbs free energy of (1) is positive. Given the negative enthalpy of reaction (1) from DFT, the Gibbs' free energy of (1) becomes less negative with increasing temperature, gradually shifting (1) towards the left hand side where $\mathrm{O}_{i}$ leaves the lattice to form $\mathrm{O}_{2}$ molecules. The equilibrium concentration of $\mathrm{O}_{\mathrm{i}}$ hence decreases with increasing temperature, in line with the results in Fig. 1, but in contrast to entropy stabilized oxygen vacancies which have a positive enthalpy of formation under conditions where the oxide is stable. In analogy to brownmillerite $\mathrm{AB}_{2} \mathrm{O}_{5}$ and perovskite $\mathrm{ABO}_{3}, \mathrm{O}_{\mathrm{i}}$ in $\mathrm{YMnO}_{3}$ could be considered filled oxygen vacancies in the hypothetical compound $\mathrm{Y}_{3} \mathrm{Mn}_{3} \mathrm{O}_{10}$. With $\mathrm{Y}_{3} \mathrm{Mn}_{3} \mathrm{O}_{10}$ as the reference state, vacant $\mathrm{O}_{\mathrm{i}}$ sites would be oxygen vacancies, and thus conform to conventional point defect thermodynamics with positive enthalpies and entropies of formation. We note that the enthalpy of formation for $\mathrm{O}_{i}$ is expected to decrease progressively with increasing oxygen excess $\delta$ due to $\mathrm{O}_{\mathrm{i}}-\mathrm{O}_{\mathrm{i}}$ repulsion and a gradual loss of the driving force for oxidation.

Migration of $\mathbf{O}_{\mathbf{i}}$. To investigate the ionic mobility of interstitial oxygen, migration paths for $\mathrm{O}_{\mathrm{i}}$ between two stable positions were investigated by NEB calculations. Simple interstitial migration, with a calculated energy barrier of almost $6 \mathrm{eV}$ (Supplementary Fig. 8a), is not likely to occur. In the interstitialcy mechanism, $\mathrm{O}_{\mathrm{i}}$ pushes an adjacent planar oxygen into a neighbouring interstitial 


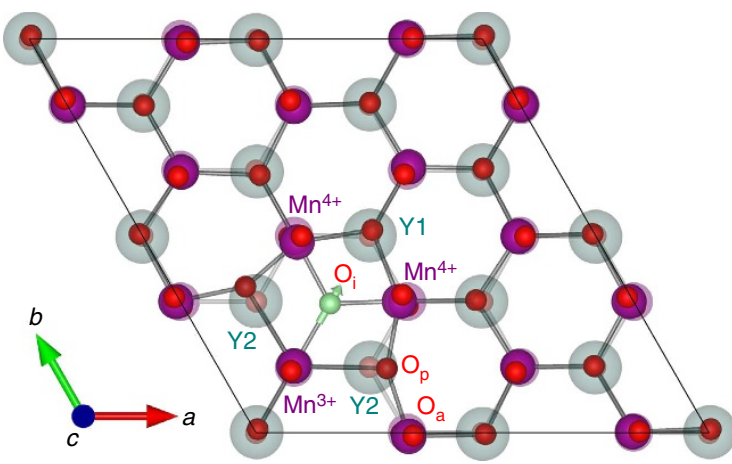

C

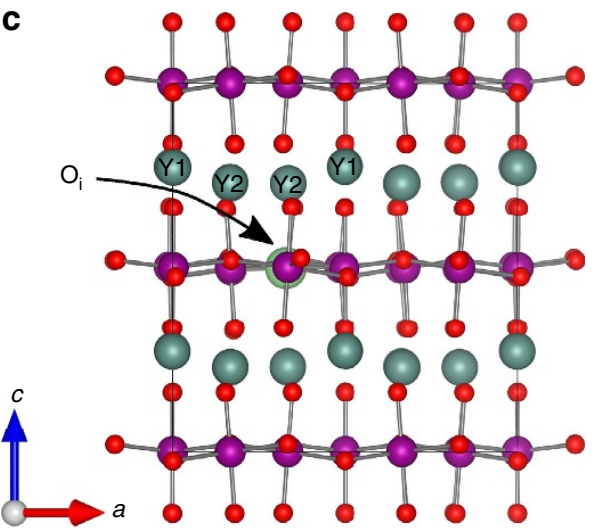

b
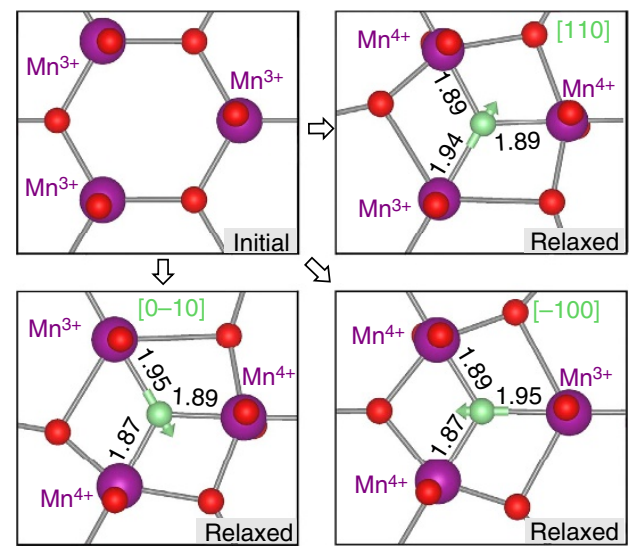

d

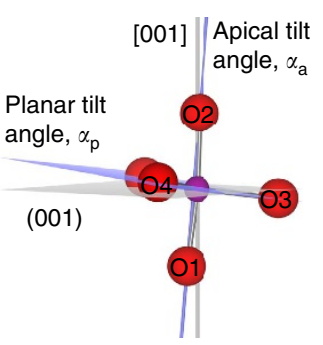

e

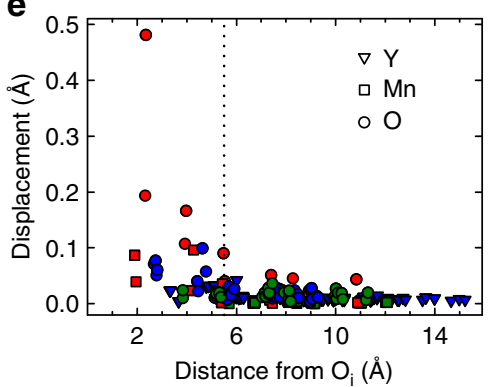

Figure 3 | Structural distortions and screening length. (a) Relaxed geometry of the (002) plane of a $2 \times 2 \times 1$ supercell of $\mathrm{YMnO}_{3}$ with the perfect structure faded in the background for comparison. Y atoms above the (002) $\mathrm{Mn}-\mathrm{O}$ plane are projected onto the plane. (b) Unrelaxed structure in upper left panel compared with relaxed structures around $\mathrm{O}_{i}$ in the three triple-well positions around the $2 / 3,2 / 3,1 / 2$ position. The green arrows on $\mathrm{O}_{i}$ indicate the displacement towards the two $\mathrm{Mn}^{4}+$. Bond lengths between $\mathrm{O}_{i}$ and $\mathrm{Mn}$ are given in $\AA$. (c) $\mathrm{YMnO}_{3}$ with $\mathrm{O}_{i}$ (green sphere in the (002) Mn-O layer) viewed along the b-direction. (d) Apical and planar tilting angles of the trigonal bipyramids. $\mathrm{O} 1$ and $\mathrm{O} 2$; and $\mathrm{O} 3$ and $\mathrm{O} 4$ being apical and planar oxygens, respectively. (e) Displacements of ions with respect to the perfect structure as a function of distance from $\mathrm{O}_{\mathrm{i}}$. The red symbols represent atoms in the same $\mathrm{Mn}-\mathrm{O}$ layer as $\mathrm{O}_{i}$ is positioned, the blue symbols represent the apical oxygens and the yttrium atoms closest to the $\mathrm{Mn}-\mathrm{O}$ layer with $\mathrm{O}_{\mathrm{i}}$, and the green symbols show atoms in the adjacent $\mathrm{Mn}-\mathrm{O}$ layers. The structural screening length is indicated by a vertical dotted line.

site, subsequently taking up a regular planar oxygen lattice position itself. The maximum energy along the minimum energy path is found when the distance between $\mathrm{O}_{\mathrm{i}}$ and planar lattice oxygen goes through a minimum. When $\mathrm{O}_{i}$ nudges a regular oxygen at an $\mathrm{O} 3$ site, (Fig. 4b) an energy barrier of $0.48 \mathrm{eV}$ was found (Fig. 4c). A similar mechanism where $\mathrm{O}_{i}$ nudges a regular oxygen at an $\mathrm{O} 4$ site resulted in a higher energy barrier of $0.62 \mathrm{eV}$ (See Supplementary Fig. 8b,c for effect of magnetic order and Hubbard $U$ ). This is explained by Y1 being closer to the moving $\mathrm{O} 3$ (which is a trimerization centre) and $\mathrm{O}_{\mathrm{i}}$ in path 2 compared with the corresponding distance between $\mathrm{Y} 2$ and the moving $\mathrm{O} 4$ and $\mathrm{O}_{\mathrm{i}}$ in path 1 (Fig. 4b) (See Supplementary Fig. 8e and for illustration of bond lengths). Migration through path 2 will therefore be aided by Y1 bonding to the two moving oxygen atoms, which is supported by the electronic density of states in Fig. 5d. An energy barrier was also estimated from the experimental TGA data by plotting time to oxidation as a function of annealing time in $\mathrm{O}_{2}$ atmosphere, as shown in Supplementary Figure 8d. The Arrhenius-type relation gave an energy barrier of $0.55 \pm 0.21 \mathrm{eV}$, in agreement with our DFT calculated values. For comparison, oxygen transport through vacancy diffusion in $\mathrm{ABO}_{3}$ perovskites has an energy barrier in the range of $0.5-2.8 \mathrm{eV}$ (ref. 26). See Supplementary Note 4 for more details about the migration barriers.
Functional properties. We now address the impact of $\mathrm{O}_{\mathrm{i}}$ on the electronic properties. The introduction of $\mathrm{O}_{\mathrm{i}}$ between three Mn (Fig. 3a) gives three edge-sharing octahedra. As these octahedra are strongly distorted, the crystal field experienced by the d-electrons of $\mathrm{Mn}$ coordination $\mathrm{O}_{\mathrm{i}}$ does not change significantly compared with the trigonal bipyramidal crystal field in the perfect crystal. The most obvious change in electronic structure, in Fig. 5, caused by $\mathrm{O}_{\mathrm{i}}$ is the appearance of a non-bonding defect state in the band gap mainly consisting of $\mathrm{Mn}^{4+} d_{x^{2} y^{2}}$ states and $\mathrm{O}_{\mathrm{i}} 2 p_{x}$ and $2 p_{y}$ states. A fraction of the occupied $\mathrm{Mn} d$ states closest to the Fermi energy is lifted above $E_{\mathrm{F}}$ on inclusion of $\mathrm{O}_{\mathrm{i}}$ as electron density is donated from $\mathrm{Mn} 3 d$ to $\mathrm{O} 2 p$. The corresponding binding states are easily seen at the bottom of the valence band. Even though charge transfer is not complete, this can formally be regarded as the oxidation of $\mathrm{Mn}^{3+}$ to $\mathrm{Mn}^{4+}$, creating holes in the valence band and $p$-type electronic conductivity. The $p$-DOS of interstitial oxygen is very similar to that of planar oxygen, which is also coordinated by only $\mathrm{Mn}$, while it differs significantly from the $p$-DOS of apical oxygen, which is coordinated by both $\mathrm{Mn}$ and Y. The highly localized character of the defect state in the band gap is characteristic for the electronic structure of an acceptor doped material, and direct experimental detection of $\mathrm{O}_{\mathrm{i}}$ in low concentrations, by for example spectroscopic techniques, would be challenging. With increasing $\mathrm{O}_{i}$ concentration, the 


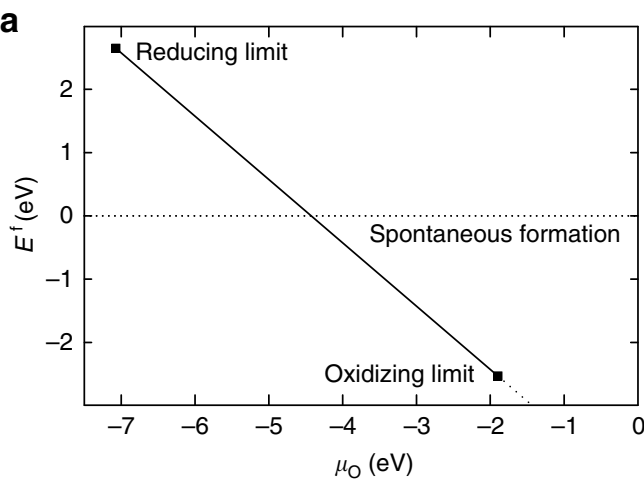

b

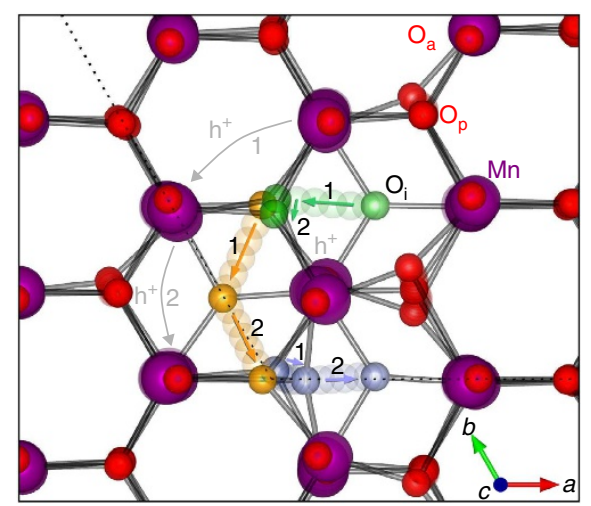

C

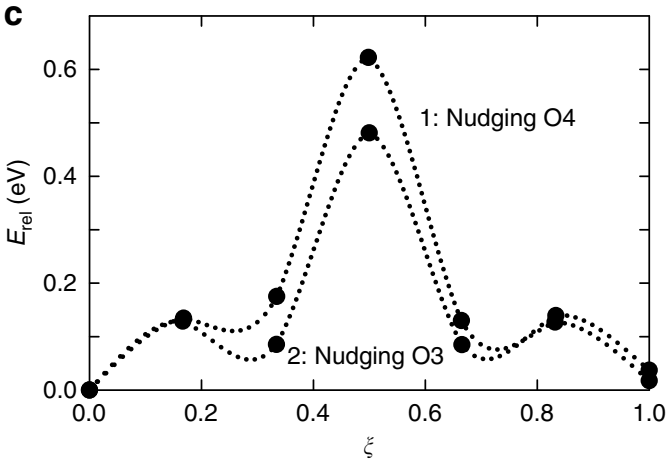

Figure 4 | Energetics and kinetics. (a) Defect formation energy of $O_{i}$ in a $2 \times 2 \times 1$ supercell of $\mathrm{YMnO}_{3}$ as a function of chemical potential of oxygen. (b) Subsequent migration paths of $\mathrm{O}_{\mathrm{i}}$ (green, then yellow and finally blue) through an interstitialcy mechanism nudging first a planar lattice oxygen at an $\mathrm{O} 4$ site (path 1) and then a planar lattice oxygen at an $\mathrm{O} 3$ site (path 2). The hopping of holes $\left(\mathrm{h}^{+}\right)$between $\mathrm{Mn}$ sites are shown by grey arrows. (c) The migration energy barriers for path 1 (nudging an 04) and path 2 (nudging an O3) as functions of the relative reaction coordinate, $\xi$.

defect state becomes less localized, as expected (See Supplementary Figure 9 for DOS and band structure of a 30 atom unit cell, $\mathrm{YMnO}_{3.16}$ ).

To address the impact of $\mathrm{O}_{\mathrm{i}}$ on the ferroelectric properties we calculated the spontaneous polarization $\left(P_{\mathrm{S}}\right)$ by the Berry phase method to $7.8 \mu \mathrm{C} \mathrm{cm}^{-2}$ for the perfect structure, and $7.2 \mu \mathrm{Ccm}^{-2}$ after inclusion of one $\mathrm{O}_{\mathrm{i}}$ in a 120 atom supercell. A simple point charge model gave 7.8 and $7.0 \mu \mathrm{Ccm}^{-2}$ for stoichiometric $\mathrm{YMnO}_{3}$ and oxidized $\mathrm{YMnO}_{3.04}$, respectively. Although the effect of $\mathrm{O}_{\mathrm{i}}$ on the spontaneous polarization is subtle, the charge compensating holes will raise the electrical conductivity and be detrimental to the macroscopic ferroelectric performance. However, in improper ferroelectrics like $\mathrm{YMnO}_{3}$,

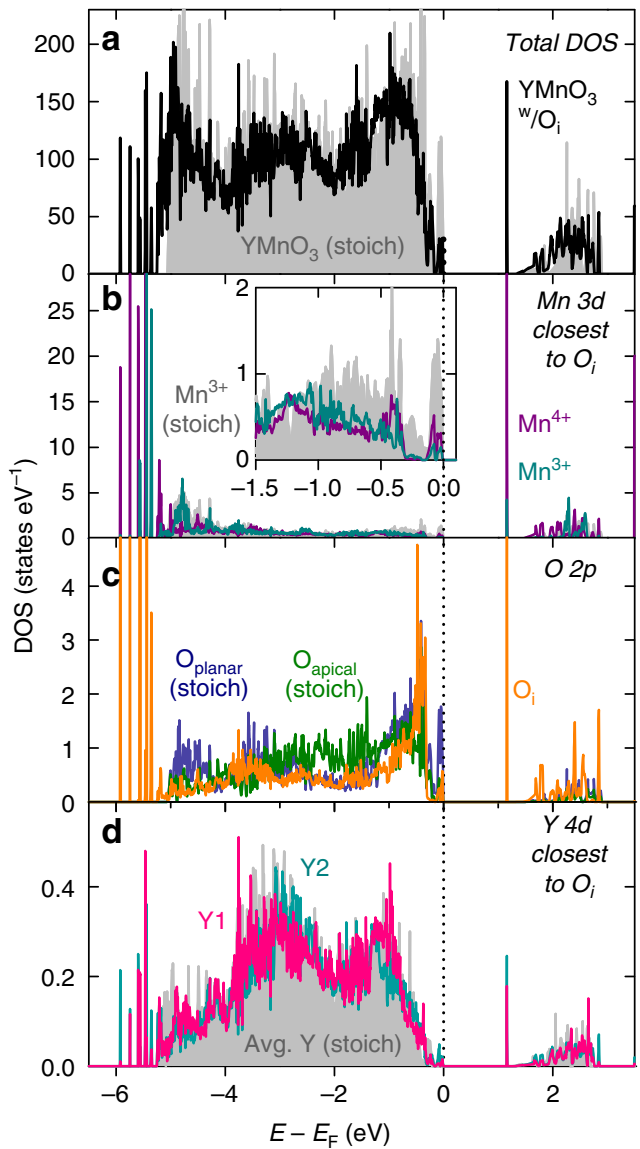

Figure 5 | Electronic structure. (a) Total electronic density of states (DOS) for a perfect 120 atom $\mathrm{YMnO}_{3}$ cell and a 121 atom cell of $\mathrm{YMnO}_{3}$ with one $\mathrm{O}_{\mathrm{i}}\left(\mathrm{YMnO}_{3.04}\right)$. (b) Atomic DOS showing the $d$ states for $\mathrm{Mn}^{3+}$ and $\mathrm{Mn}^{4+}$ coordinating $\mathrm{O}_{i}$ compared with $\mathrm{Mn}^{3}+d$ states in a stoichiometric cell. (c) Atomic DOS showing the $p$ states of $\mathrm{O}_{i}$ compared with $p$ states for apical and planar oxygens in a stoichiometric cell. (d) Atomic DOS showing the $d$ states of the closest $Y 1$ and $Y 2$ to $\mathrm{O}_{i}$ compared with the average $Y d$ states in a stoichiometric cell.

charged domain walls (DW) display anisotropic conductance due to the accumulation of mobile charge carriers ${ }^{27}$. Interstitial oxygen with formal charge -2 can screen the electrostatic field at head-to-head DWs, while charge compensating holes can screen tail-to-tail DW. Engineering point defect populations at DWs has great potential for tuning the properties of DWs as functional elements for electronics ${ }^{28-31}$.

To summarize, we have shown that interstitial oxygen is the dominating point defect in $\mathrm{YMnO}_{3}$ and the source of $p$-type electronic conductivity at ambient conditions. Interstitial oxygen is an enthalpy stabilized point defect, implying that $\mathrm{YMnO}_{3}$ is not only metastable with respect to $\mathrm{Y}_{2} \mathrm{O}_{3}$ and $\mathrm{YMn}_{2} \mathrm{O}_{5}$, in accordance with the phase diagram ${ }^{32}$, it is also metastable with respect to oxidation towards the limiting case of $\mathrm{Y}_{3} \mathrm{Mn}_{3} \mathrm{O}_{10}$. A bulk $\mathrm{YMnO}_{3}$ sample will however not oxidize completely at ambient conditions for kinetic reasons; the diffusion length is too large compared with the relatively low ionic mobility at room temperature. However, at the nanoscale, in thin films and at surfaces, oxidation will occur spontaneously also at ambient conditions. This emphasizes the necessity and potential which lies in controlling the thermal and atmospheric history of hexagonal manganite materials in order to tailor the point defect population, charge carrier concentration and physical properties. 


\section{Methods}

Experimental. Bulk $\mathrm{YMnO}_{3}$ powder was prepared by firing pressed pellets of dried and mixed $\mathrm{Y}_{2} \mathrm{O}_{3}$ and $\mathrm{MnO}_{2}$ twice for $24 \mathrm{~h}$ at $1,300{ }^{\circ} \mathrm{C}$ in air. Porous polycrystalline bars with a density of $43 \%$ were sintered from phase pure $\mathrm{YMnO}_{3}$ powder with $20 \mathrm{wt} \%$ carbon black as a pore filler for $2 \mathrm{~h}$ at $1,500^{\circ} \mathrm{C}$ in air and scanning electron micrographs of the fracture surfaces of the porous bars are given in Supplementary Fig. 10. Porous bars were used to increase the total surface area accessible to oxygen surface exchange. Four-point electrical conductivity measurements were performed on a porous polycrystalline bar in flowing $\mathrm{O}_{2}$ and $\mathrm{N}_{2}$ (ref. 33). Impedance spectroscopy measurements (Novotherm, Novocontrol Technologies) were conducted within a temperature range from 30 to $350^{\circ} \mathrm{C}$ on disc shaped samples using frequencies between $1 \mathrm{~Hz}$ and $1 \mathrm{MHz}$. Before measurement the samples were annealed in flowing oxygen resp. nitrogen for $24 \mathrm{~h}$ at $350^{\circ} \mathrm{C}$. Thermogravimetry (TGA) was done with a Netzsch STA 449C Jupiter in flowing $\mathrm{N}_{2}$ and $\mathrm{O}_{2}$ on nanoparticles with $d_{\mathrm{XRD}}=49 \pm 4 \mathrm{~nm}$ (ref. 17). Seebeck coefficients were measured at $200-500{ }^{\circ} \mathrm{C}$ in flowing $\mathrm{O}_{2}$ and $\mathrm{N}_{2}$ with a ProboStat setup (NorECs AS) on a porous bar in a vertical tubular furnace ${ }^{34}$. More details on the synthesis and characterization can be found in Supplementary Methods.

Computational. Density functional theory calculations were performed with the VASP $^{35,36}$ code and the spin polarized GGA $+U$ implementation of Dudarev ${ }^{37}$ with the PBEsol functional ${ }^{38}$. A Hubbard $U$ of $5 \mathrm{eV}$ was applied to the $\mathrm{Mn} 3 d$ orbitals in order to reproduce the experimental band gap ${ }^{39}$ and lattice parameters ${ }^{40}$. The projector augmented wave ${ }^{41}$ method was used treating $\mathrm{Y}(4 s, 4 p, 4 d, 5 s), \mathrm{Mn}(3 s, 3 p, 3 d, 4 s)$ and $\mathrm{O}(2 s, 2 p)$ as valence electrons and a plane-wave cutoff energy of $550 \mathrm{eV}$. Brillouin zone integration was done on a $\Gamma$-centered $4 \times 4 \times 2$ mesh for the 30 atom unit cell and with the number of $k$-points reduced accordingly for supercells. The non-collinear magnetic structure was approximated by a frustrated collinear antiferromagnetic (F-AFM) ${ }^{42}$ order in most of the calculations, which does not give significantly different results from calculations with the true non-collinear magnetic order. Geometry optimization was done until the forces on the ions were below $0.005 \mathrm{eV}^{-1}$ for 121 atom cells and 0.02 for 541 atom cells. The reducing and oxidizing limit for the chemical potential of oxygen varies from -7.1 to $-1.9 \mathrm{eV}$. The defect formation energy of $\mathrm{O}_{\mathrm{i}}$ was $0.03 \mathrm{eV}$ lower in a 121 atom cell than in a 541 atom cell (See Supplementary Fig. 11). Bader analysis ${ }^{43}$ gave charges of +1.90 and +1.73 for $\mathrm{Mn}^{4+}$ and $\mathrm{Mn}^{3+}$, respectively. For the nudged elastic band ${ }^{44}$ (NEB) calculations we used five intermediate images for the $\mathrm{O}_{\mathrm{i}}$ migration path and a Hubbard $U=5 \mathrm{eV}$ was applied to reproduce the nature of the localized electron holes (comparison with $U=0 \mathrm{eV}$ can be found in the Supplementary Fig. 8b). A frustrated antiferromagnetic (F-AFM) order was also used for the calculation of the energy barriers. Comparison between A-type AFM and F-AFM magnetic order is given in Supplementary Fig. $8 c$, and was set so that no spin flipping on the Mn ions occurred during the migration of $\mathrm{O}_{\mathrm{i}}$ in order to isolate the effect of pure migration. The ferroelectric polarization was calculated with the Berry phase method ${ }^{45}$. VESTA was used for visualizing structures ${ }^{46}$.

Data availability. The data that support the findings of this study are available from the authors on reasonable request.

\section{References}

1. Bagotsky, V. S. Fuel Cells: Problems and Solutions (Wiley, 2009).

2. Tarascon, J.-M. \& Armand, M. Issues and challenges facing rechargeable lithium batteries. Nature 414, 359-367 (2001).

3. Waser, R., Dittmann, R., Staikov, G. \& Szot, K. Redox-based resistive switching memories-nanoionic mechanisms, prospects, and challenges. Adv. Mater. 21, $2632-2663$ (2009)

4. Aggarwal, S. \& Ramesh, R. Point defect chemistry of metal oxide heterostructures. Annu. Rev. Mater. Sci. 28, 463-499 (1998).

5. Grande, T., Tolchard, J. R. \& Selbach, S. M. Anisotropic thermal and chemical expansion in $\mathrm{Sr}$-substituted $\mathrm{LaMnO}_{3+\delta}$ : implications for chemical strain relaxation. Chem. Mater. 24, 338-345 (2012).

6. Kalinin, S. V. \& Spaldin, N. A. Functional ion defects in transition metal oxides. Science 341, 858-859 (2013).

7. Kalinin, S. V., Borisevich \& Fong, D. Beyond condensed matter physics on the nanoscale: the role of ionic and electrochemical phenomena in the physical functionalities of oxide materials. ASC Nano 6, 10423-10437 (2012).

8. Gibbs, A. S., Knight, K. S. \& Lightfoot, P. High-temperature phase transitions of hexagonal $\mathrm{YMnO}_{3}$. Phys. Rev. B 83, 094111 (2011).

9. Van Aken, B. B., Palstra, T. T. M., Filippetti, A. \& Spaldin, N. A. The origin of ferroelectricity in magnetoelectric $\mathrm{YMnO}_{3}$. Nat. Mater. 3, 164-170 (2004)

10. Fiebig, M., Lottermoser, T., Frohlich, D., Goltsev, A. V. \& Pisarev, R. V. Observation of coupled magnetic and electric domains. Nature 419, 818-820 (2002)

11. Overton, A. J., Best, J. L., Saratovsky, I. \& Hayward, M. Influence of topotactic reduction on the structure and magnetism of the multiferroic $\mathrm{YMnO}_{3}$. Chem. Mater. 21, 4940-4948 (2009).
12. Du, Y. et al. Manipulation of domain wall mobility by oxygen vacancy ordering in multiferroic $\mathrm{YMnO}_{3}$. Phys. Chem. Chem. Phys. 15, 20010-20015 (2013).

13. Cheng, S., Deng, S. Q., Zhao, Y. G., Sun, X. F. \& Zhu, J. Correlation between oxygen vacancies and sites of $\mathrm{Mn}$ ions in $\mathrm{YMnO}_{3}$. Appl. Phys. Lett. 106, 062905 (2015).

14. Remsen, S. \& Dabrowski, B. Synthesis and oxygen storage capacities of hexagonal $\mathrm{Dy}_{1-x} \mathrm{Y}_{x} \mathrm{MnO}_{3+\delta}$. Chem. Mater. 23, 3818-3827 (2011).

15. Parkkima, O., Malo, S., Hervieu, M., Rautama, E.-L. \& Karppinen, M. New $R \mathrm{MnO}_{3+\delta}(R=\mathrm{Y}, \mathrm{Ho} ; \delta \approx 0.35)$ phases with modulated structure. J. Solid State Chem. 221, 109-115 (2015).

16. Roosmalen, J. A. \& Cordfunke, E. H. The defect chemistry of $\mathrm{LaMnO}_{3+\delta}$ : 4. defect model for $\mathrm{LaMnO}_{3}$. J. Solid State Chem. 110, 109-112 (1994).

17. Bergum, K. et al. Synthesis, structure and magnetic properties of nanocrystalline $\mathrm{YMnO}_{3}$. Dalt. Trans. 40, 7583-7589 (2011).

18. Tomczyk, M., Vilarinho, P. M., Moreira, A. \& Almeida, A. High temperature dielectric properties of $\mathrm{YMnO}_{3}$ ceramics. J. Appl. Phys. 110, 064116 (2011).

19. Ren, P., Fan, H. \& Wang, X. Bulk conduction and nonlinear behaviour in multiferroic $\mathrm{YMnO}_{3}$. Appl. Phys. Lett. 103, 152905 (2013).

20. Bogusz, A. et al. Resistive switching in polycrystalline $\mathrm{YMnO}_{3}$ thin films. AIP Adv. 4, 107135 (2014).

21. Van Aken, B. B., Bos, J.-W. G., de Groot, R. A. \& Palstra, T. T. M. Asymmetry of electron and hole doping in $\mathrm{YMnO}_{3}$. Phys. Rev. B 63, 125127 (2001).

22. Moure, C., Villegas, M., Fernandez, J. F., Tartaj, J. \& Duran, P. Phase transition and electrical conductivity in the system $\mathrm{YMnO}_{3}-\mathrm{CaMnO}_{3}$. J. Mater. Sci. 34, 2565-2568 (1999).

23. Balamurugan, C. \& Lee, D. W. Perovskite hexagonal $\mathrm{YMnO}_{3}$ nanopowder as p-type semiconductor gas sensor for $\mathrm{H}_{2} \mathrm{~S}$ detection. Sens. Actuators B: Chem. 221, 857-866 (2015).

24. Ma, Y. et al. Investigation of magnetic, dielectric and electrical properties of Ce-substituted $\mathrm{YMn}_{0.8} \mathrm{Fe}_{0.2} \mathrm{O}_{3}$ multiferroic ceramics. J. Mater. Sci.: Mater. Electron. 26, 398-404 (2015).

25. Zhang, S. B. \& Northrup, J. E. Chemical potential dependence of defect formation energies in GaAs: application to Ga self-diffusion. Phys. Rev. Lett. 67, 2339-2342 (1991).

26. Chroneos, A., Vovk, R. V., Goulatis, I. L. \& Goulatis, L. I. Oxygen transport in perovskite and related oxides: A brief review. J. Alloy. Compd. 494, 190-195 (2010).

27. Meier, D. et al. Anisotropic conductance at improper ferroelectric domain walls. Nat. Mater. 11, 284-288 (2012).

28. Griffin, S. M. et al. Scaling behavior and beyond equilibrium in the hexagonal manganites. Phys. Rev. X 2, 041022 (2012).

29. Choi, T. et al. Insulating interlocked ferroelectric and structural antiphase domain walls in multiferroic $\mathrm{YMnO}_{3}$. Nat. Mater. 9, 253-258 (2010).

30. Meier, D. Functional domain walls in multiferroics. J. Phys.: Condens. Matter 27, 463003 (2015).

31. Kumagai, Y. \& Spaldin, N. A. Structural domain walls in polar hexagonal manganites. Nat. Commun. 4, 1540 (2013).

32. Chen, M., Hallstedt, B. \& Gauckler, L. J. Thermodynamic assessment of the Mn-Y-O system. J. Alloy. Compd. 393, 114-121 (2005).

33. Selbach, S. M., Tybell, T., Einarsrud, M.-A. \& Grande, T. High-temperature semiconducting cubic phase of $\mathrm{BiFe}_{0.7} \mathrm{Mn}_{0.3} \mathrm{O}_{3+\delta}$. Phys. Rev. B 79, 214113 (2009).

34. Wefring, E. T., Einarsrud, M. A. \& Grande, T. Electrical conductivity and thermopower of $(1-x) \mathrm{BiFeO}_{3}-x \mathrm{Bi}_{0.5} \mathrm{~K}_{0.5} \mathrm{TiO}_{3}(x=0.1,0.2)$ ceramics near the ferroelectric to paraelectric phase transition. Phys. Chem. Chem. Phys. 17, 9420-9428 (2015).

35. Kresse, G. \& Furthmüller, J. Efficient iterative schemes for $a b$ initio total-energy calculations using a plane-wave basis set. Phys. Rev. B 54, 11169-11186 (1996)

36. Kresse, G. \& Joubert, D. From ultrasoft pseudopotentials to the projector augmented-wave method. Phys. Rev. B 59, 1758-1775 (1999).

37. Dudarev, S. L., Botton, G. A., Savrasov, S. Y., Humphreys, C. J. \& Sutton, A. P. Electron-energy-loss spectra and the structural stability of nickel oxide: An LSDA + U study. Phys. Rev. B 57, 1505-1509 (1998).

38. Perdew, J. P. et al. Restoring the density-gradient expansion for exchange in solids and surfaces. Phys. Rev. Lett. 100, 136406 (2008).

39. Degenhardt, C., Fiebig, M., Fröhlich, D., Lottermoser, T. \& Pisarev, R. V. Nonlinear optical spectroscopy of electronic transitions in hexagonal manganites. Appl. Phys. B 73, 139-144 (2001).

40. Van Aken, B. B., Meetsma, A. \& Palstra, T. T. M. Hexagonal $\mathrm{YMnO}_{3}$. Acta Crystallogr. C 57, 230-232 (2001).

41. Blöchl, P. E. Projector augmented-wave method. Phys. Rev. B 50, 17953-17979 (1994).

42. Medvedeva, J. E., Anisimov, V. I., Korotin, M. A., Mryasov, O. N. \& Freeman, A. J. The effect of Coulomb correlation and magnetic ordering on the electronic structure of two hexagonal phases of ferroelectromagnetic $\mathrm{YMnO}_{3}$. J. Phys.: Condens. Matter 12, 4947-4958 (2000). 
43. Henkelman, G., Arnaldsson, A. \& Jónsson, H. A fast and robust algorithm for Bader decomposition of charge density. Comput. Mater. Sci. 36, 354-360 (2006).

44. Henkelman, G., Uberuaga, B. P. \& Jonsson, H. A climbing image nudged elastic band method for finding saddle points and minimum energy paths. J. Chem. Phys. 113, 9901-9904 (2000).

45. Spaldin, N. A. A beginner's guide to the modern theory of polarization. J. Solid State Chem. 195, 2-10 (2012).

46. Momma, K. \& Izumi, F. VESTA: a three-dimensional visualization system for electronic and structural analysis. J. Appl. Crystallogr. 41, 653-658 (2008).

\section{Acknowledgements}

The Research Council of Norway (FRINATEK project no.231430/F20) and Norwegian University of Science and Technology (NTNU) are acknowledged for financial support. Computational resources were provided by NOTUR (The Norwegian Metacenter for High Performance Computing) through the project NN9264K and the Euler cluster at ETH, Zürich. Dr Kristin Bergum is acknowledged for preparing the nanoparticles for TGA.

\section{Author contributions}

S.H.S. and G.H.O. performed DFT calculations; E.T.W. conducted thermoelectric measurements; S.K.N. and N.H.G. performed DC conductivity and prepared bulk samples; J.G. and S.H.S. performed AC impedance measurements; S.M.S. performed TGA; S.M.S. and N.H.G. performed HTXRD; S.H.S., T.T. and S.M.S. wrote the paper; all authors

discussed the results; S.M.S. conceived the project; T.T. and S.M.S. supervised the project.

\section{Additional information}

Supplementary Information accompanies this paper at http://www.nature.com/ naturecommunications

Competing financial interests: The authors declare no competing financial interests.

Reprints and permission information is available online at http://npg.nature.com/ reprintsandpermissions/

How to cite this article: Skjærvø, S. H. et al. Interstitial oxygen as a source of $p$-type conductivity in hexagonal manganites. Nat. Commun. 7, 13745 doi: 10.1038/ ncomms13745 (2016).

Publisher's note: Springer Nature remains neutral with regard to jurisdictional claims in published maps and institutional affiliations.

\section{(c) (i)}

This work is licensed under a Creative Commons Attribution 4.0 International License. The images or other third party material in this article are included in the article's Creative Commons license, unless indicated otherwise in the credit line; if the material is not included under the Creative Commons license, users will need to obtain permission from the license holder to reproduce the material. To view a copy of this license, visit http://creativecommons.org/licenses/by/4.0/

(C) The Author(s) 2016 